OPEN ACCESS

Edited by:

Ricardo M. Leao,

University of São Paulo, Brazil

Reviewed by:

Todd Sacktor,

Suny Downstate Medical Center,

United States

Yi Zhong,

Tsinghua University, China

*Correspondence:

Ping Zheng

pzheng@shmu.edu.cn

Bin Lai

laibin@fudan.edu.cn

†Lead Contact

Specialty section:

This article was submitted to Molecular Signalling and Pathways,

a section of the journal

Frontiers in Molecular Neuroscience

Received: 04 June 2021

Accepted: 27 July 2021

Published: 31 August 2021

Citation:

Cui $D$, Jiang $X$, Chen $M$, Sheng $H$, Shao $D$, Yang $L$, Guo $X$, Wang $Y$, Lai $B$ and Zheng $P$ (2021) Activation of Rac1 Has an Opposing Effect on Induction and Maintenance of Long-Term Potentiation in Hippocampus by

Acting on Different Kinases. Front. Mol. Neurosci. 14:720371. doi: 10.3389/fnmol.2021.720371

\section{Activation of Rac1 Has an Opposing Effect on Induction and Maintenance of Long-Term Potentiation in Hippocampus by Acting on Different Kinases}

\author{
Dongyang Cui ${ }^{1}$, Xiaodong Jiang ${ }^{1}$, Ming Chen ${ }^{1}$, Huan Sheng ${ }^{1}$, Da Shao ${ }^{1}$, Li Yang ${ }^{1}$, \\ Xinli Guo ${ }^{1}$, Yingqi Wang ${ }^{1}$, Bin Lai ${ }^{1 *}$ and Ping Zheng ${ }^{1,2,3 * \dagger}$
}

${ }^{1}$ State Key Laboratory of Medical Neurobiology, Institutes of Brain Science, MOE Frontier Center for Brain Science, School of Basic Medical Sciences, Fudan University, Shanghai, China, ${ }^{2}$ Department of Neurology of Zhongshan Hospital, Fudan University, Shanghai, China, ${ }^{3}$ Department of Pharmacology of Medical College of China Three Gorges University, Yichang, China

Rac1 is a small GTPase of the Rho family. A previous study showed that the activation of Rac1 had an opposing effect on induction and maintenance of long-term potentiation (LTP) in the hippocampus. However, the molecular mechanism underlying this opposing effect remains to be addressed. In the present work, we find that the activation of Rac1 during the induction of LTP leads to an activation of $\mathrm{PKC} \iota / \lambda$ by phosphatidylinositol-3-kinase (PI3K), whereas the activation of Rac1 during the maintenance of LTP leads to the inhibition of PKM by LIM_kinase (LIMK) in the hippocampus. This result suggests that during different stages of LTP, the activation of Rac1 can modulate different signaling pathways, which leads to an opposing effect on the induction and maintenance of LTP in the hippocampus.

Keywords: Rac1, long term potentiation, PI3K, LIMK, PKC $\_/ \lambda, \mathrm{PKM} \zeta$

\section{INTRODUCTION}

Small GTPases are important signaling molecules in neurons. One of the best characterized subfamilies of the small GTPases is the Rho family, which includes Rac, Cdc42, and Rho (Hall, 2005). Among them, Racl has been reported to be involved in morphological plasticity in the hippocampus. Rac1 could induce spine morphogenesis and synapse formation in the hippocampus (Luo, 2000; Tolias et al., 2005). In addition, Rac1 also participates in functional plasticity in the hippocampus. Martinez and Tejada-Simon (2011) reported that the induction of longterm potentiation (LTP) in the hippocampus was coupled with the activation of Racl and the inhibition of Racl suppressed the induction of LTP in a dose-dependent manner (Martinez and Tejada-Simon, 2011). Interestingly, during the maintenance phase of LTP in the hippocampus, it appeared that the activation of Rac1 had an opposing effect on LTP. Liu et al. reported that the application of an adeno-associated virus that carried transgene to activate Racl during the maintenance phase of LTP resulted in an accelerated LTP decay in the hippocampus (Liu et al., 2016). However, the molecular mechanism underlying the opposing effect of the activation of Racl on the induction and maintenance of LTP in the hippocampus remains to be addressed. 
Typical downstream signal transduction pathway of the activation of Rac1 includes two steps: first, the activation of p21-activated kinase (Pak); second, the Pak-induced activation of LIM-domain-containing protein kinase (LIM-kinase), which subsequently phosphorylates and inhibits cofilin, an actin depolymerization factor, thus inducing actin polymerization (Luo, 2000). However, it is hard to explain the opposing effect of the activation of Racl on the induction and maintenance of LTP using this typical downstream signal transduction pathway of Racl.

It has been known that numerous signaling molecules have been involved in the induction and maintenance of LTP (Baltaci et al., 2019). Among them, Wang et al. (2016) reported that phosphorylated protein kinase $\mathrm{C}$ iota/lambda $(\mathrm{pPKC} / / \lambda)$ showed a marked increase during the induction phase of LTP but returned to the control level during the maintenance phase of LTP, whereas PKM $\zeta$ increased significantly only during the maintenance phase of LTP (Wang et al., 2016). Using a recombinant adeno-associated virus (rAAV2/8) expressing small hairpin RNA (shRNA) that targeted the gene of either $\mathrm{PKC} \iota / \lambda$ or $\mathrm{PKM} \zeta$, it was found that the knockdown of $\mathrm{PKC} \iota / \lambda$ produced a reduction in the early expression of LTP during the induction phase, whereas the knockdown of $\mathrm{PKM} \zeta$ disrupted only the late phase of LTP during the maintenance phase (Wang et al., 2016). These pieces of evidence suggest that the activation of $\mathrm{PKC} / \lambda$ played an important role in the induction of LTP, while the activation of PKM $\zeta$ played a key role in the maintenance of LTP. Therefore, we proposed a hypothesis that Rac1 might have an opposing effect on $\mathrm{PKC} / / \lambda$ and $\mathrm{PKM} \zeta$, thus producing an opposing effect on the induction and maintenance of LTP in the hippocampus. To test this hypothesis, using electrophysiological method combined with the Western blotting and pharmacological approaches, we studied the role of the activation of Racl during the induction and maintenance phases of LTP in the hippocampus and further explored the downstream signaling pathways of Racl activation during the induction and maintenance phases of LTP.

\section{RESULTS}

\section{Activation of Rac1 Has an Opposing Effect on the Induction and Maintenance of LTP in Hippocampal CA1}

Long-term potentiation (LTP) is generally divided into at least two distinct phases: the induction phase and the maintenance phase (Baltaci et al., 2019). To evaluate the role of Rac1 in the induction of LTP, we first detected whether Racl was activated during the induction phase of LTP. Rats were divided into four groups: one group was the control group where Hippocampal CA1 slices were not given high-frequency stimulation (HFS), and the other three groups were divided into 1-min group, 10-min group, and 30-min group based on the time duration after giving HFS. The result showed that the level of the activation state of Rac1 (Rac1-GTP) was significantly increased at 10 min after HFS (one-way ANOVA, $F_{(3,8)}=63.24$, control group, $0.19 \pm$ $0.029, n=3$; 10-min group, $0.84 \pm 0.033, n=3 ; P<0.0001$; Figure 1A), but returned to control level at $30 \mathrm{~min}$ after HFS (one-way ANOVA, 30-min group, $0.30 \pm 0.055, n=3$, vs. control group; $P=0.1974$; Figure 1A). This result suggests that LTP induction is associated with a transient activation of Racl. We then studied the role of Racl activation in LTP induction by examining the influence of Rac1-specific inhibitor NSC23766 (Martinez and Tejada-Simon, 2011) on the LTP induction. Rats were divided into two groups: control group where artificial cerebrospinal fluid (ACSF) was applied at $30 \mathrm{~min}$ before HFS, and NSC23766 group where NSC23766 $(100 \mu \mathrm{M})$ was applied at $30 \mathrm{~min}$ before HFS. The result showed that after the application of NSC23766, Rac1 activation by HFS at 10 min after HFS was significantly inhibited when compared with the control group (unpaired $t$-test, $t_{(4)}=4.893$, control group, $0.85 \pm 0.068, n=3$; NSC23766 group, $0.49 \pm 0.029, n=3 ; P=0.0081$; Figure 1B), and LTP induction by HFS was also significantly inhibited by NSC23766 (unpaired $t$-test, $t_{(10)}=5.592$, control group, $146.7 \pm$ $4.1 \%, n=6$; NSC23766 group, $108.3 \pm 8.8 \%, n=6 ; P=0.0027$; Figure $1 \mathrm{C}$ ). These results suggest that Racl activation contributes significantly to LTP induction.

We also evaluated the contribution of Racl activation to LTP maintenance by examining the influence of Rac1-specific inhibitor NSC23766 on the LTP maintenance. NSC23766 was applied $10 \mathrm{~min}$ after the last HFS. Result showed that LTP maintenance was unaffected after the application of NSC23766 (unpaired $t$-test, $t_{(10)}=0.3290$, control group, $139.0 \pm 5.9 \%$, $n=6$; NSC23766 group, $138.1 \pm 7.7 \%, n=6, P=0.7489$; Figure 2A). This result suggests that Racl activation does not contribute to LTP maintenance. Interestingly, when we applied Rac1 agonist CN04 (Jiang et al., 2016) during the maintenance phase of LTP, CN04 induced an accelerated decay of LTP. The rats were divided into two groups: control group where ACSF was applied and CN04 group where CN04 (424 nM) was applied. The top panel of Figure 2B shows that in a normal brain slice containing the CA1 region, 90 min treatment with CN04 could significantly increase the level of Rac1-GTP (unpaired $t$-test, $t_{(4)}=7.044$, control group, $0.56 \pm 0.047, n=3$; CN04 group, $1.13 \pm 0.065, n=3 ; P=0.0021$; Top panel of Figure 2B). The middle and bottom panels of Figure $2 \mathbf{B}$ show the effect of CN04 on LTP maintenance and baseline of field excitatory postsynaptic potentials (fEPSPs). We could see that CN04 treatment resulted in an accelerated LTP decay during the maintenance phase (unpaired $t$-test, $t_{(10)}=5.831$, control group, $123.2 \pm 6.4 \%$, $n=6$; CN04 group, $69.1 \pm 6.7 \%, n=6 ; P=0.0002$; The middle panel of Figure 2B), whereas the treatment had no influence on baseline of fEPSPs (unpaired $t$-test, $t_{(10)}=5.592,30 \mathrm{~min}$ control group, $97.3 \pm 4.5 \%, n=6 ; 210$ min CN04 group, $107.0 \pm 5.8 \%, n$ $=6 ; P=0.2239$; The bottom panel of Figure 2B). CN04 is not a specific activator of Rac1 agonist, as it also activates CDC42 and RhoA. So the influence of CN04 on LTP may be due to Rac1, CDC42, or RhoA. To confirm the CN04-induced decrease in LTP maintenance was mediated by Rac1 activation, the Pak1, a specific downstream molecular of Racl pathway, was inhibited by IPA-3 before CN04 treatment. The rats were divided into two groups: CN04 group where CN04 $(424 \mathrm{nM})$ was applied, and IPA-3 + CN04 group where the Pak1 inhibitor IPA-3 (100 $\mu \mathrm{M})$ and CN04 were co-applied. Supplementary Figure 1 shows that Pak1 inhibitor IPA-3 could reverse CN04-induced decrease in LTP maintenance (unpaired $t$-test, $t_{(10)}=3.270$, CN04 group, 
A
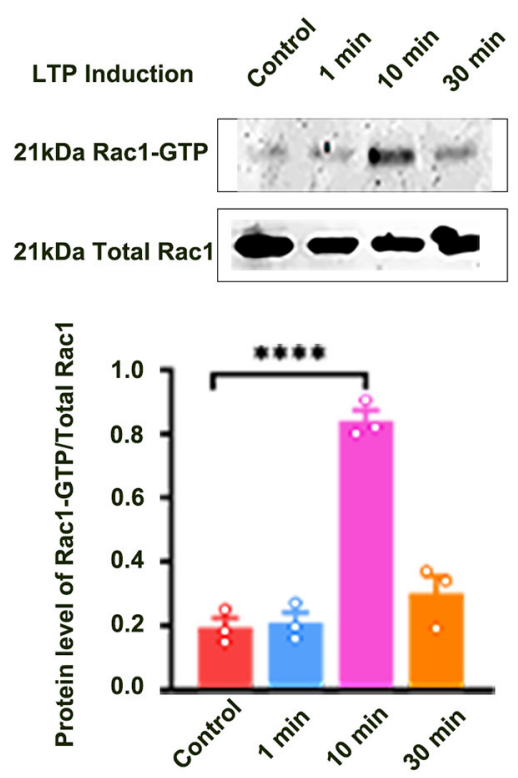

B
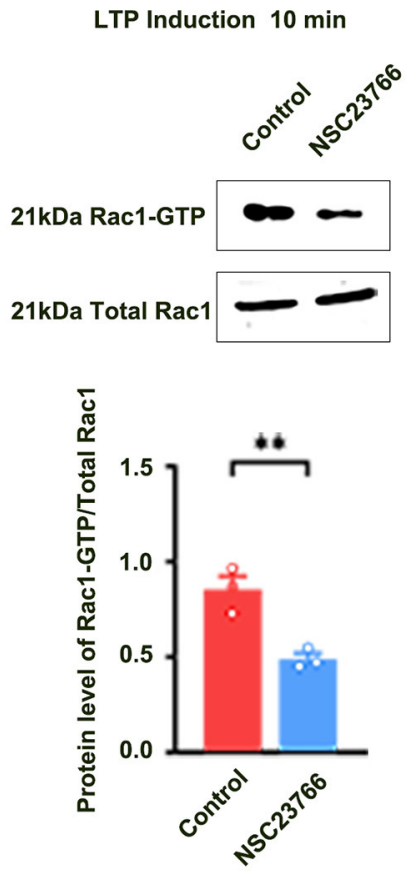

c

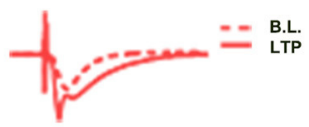

$=$ B.L.

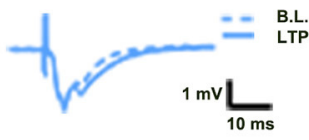

LTP
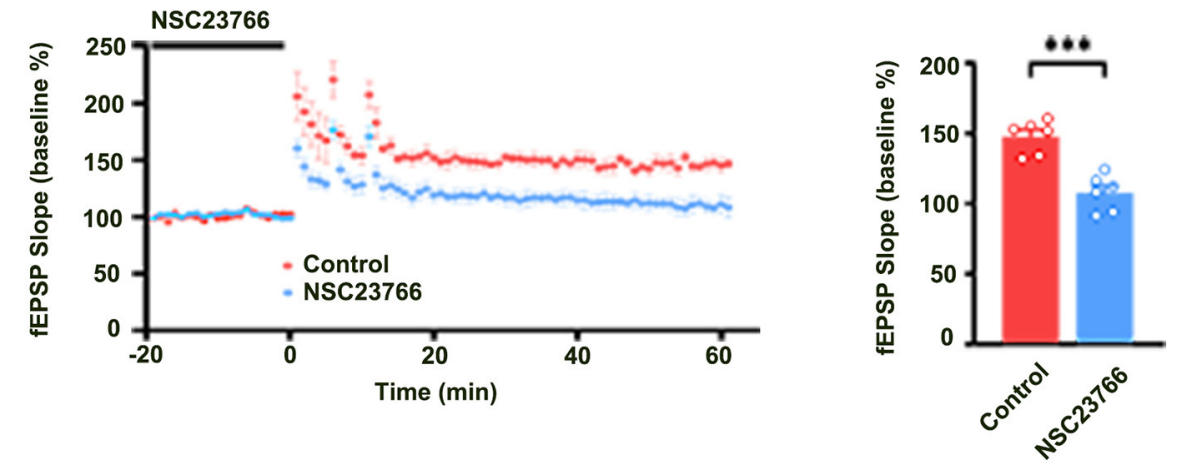

FIGURE 1 | The role of Rac1 in LTP induction. (A) Influence of three trains of HFS on Rac1 activation (one-way ANOVA, ${ }^{* \star * \star} P<0.0001, n=3$ in each group). (B) Influence of Rac1 antagonist NSC23766 $(100 \mu \mathrm{M})$ on Rac1 activation at 10 min after HFS (unpaired $t$-test, ${ }^{\star \star} P<0.01, n=3$ in each group). (C) Influence of Rac1 antagonist NSC23766 (100 $\mu \mathrm{M}$ ) on LTP induction (unpaired $t$-test, ${ }^{* \star *} P<0.001, n=6$ in each group). Top: Typical fEPSP trace at baseline and during LTP recordings. Left: LTP was recorded for $1 \mathrm{~h}$ after three trains of HFS. Right: Average fEPSP slope during the last $15 \mathrm{~min}$ of the LTP recording. All data are shown as mean \pm SEM.

$69.1 \pm 6.7 \%, n=6$; IPA-3 + CN04 group, $116.4 \pm 7.7 \%, n$ $=6 ; P=0.0084$; The bottom panel of Figure $2 \mathrm{~B}$ ), suggesting that CN04-induced decrease in LTP maintenance was related to Racl activation. These results suggest that the activation of Rac1 during the maintenance phase accelerates LTP decay.

\section{Activation of Rac1 During the Induction Phase of LTP Results in Activation of $\mathrm{PKC} \iota / \lambda$ Through PI3K Pathway in the Hippocampus}

Previous study showed that $\mathrm{PKC} / \lambda$ was activated during the induction phase of LTP and the knockdown of $\mathrm{PKC} \iota / \lambda$ could inhibit LTP induction (Wang et al., 2016). However, the manner in which $\mathrm{PKC} / \lambda$ is activated during the induction phase of LTP remains to be addressed. To evaluate whether Rac1 activation was an upstream mechanism of the activation of $\mathrm{PKC} / \lambda$ during the induction phase of LTP, we examined the influence of Rac1 inhibitor NSC23766 on HFS-induced activation of $\mathrm{PKC} / \lambda$. The rats were divided into three groups:one group was the control group where the slices were not given HFS, and the other two groups were divided into 10 - min group and 30-min group according to the time duration after giving HFS. Figure 3A shows HFS-induced activation of $\mathrm{PKC} \iota / \lambda$ (one-way ANOVA, $F_{(2,6)}=19.48$, control group, $0.49 \pm 0.041, n=3 ; 30$-min group, $1.06 \pm 0.090, n=3 ; P=0.0020$; Figure 3A). Figure 3B 


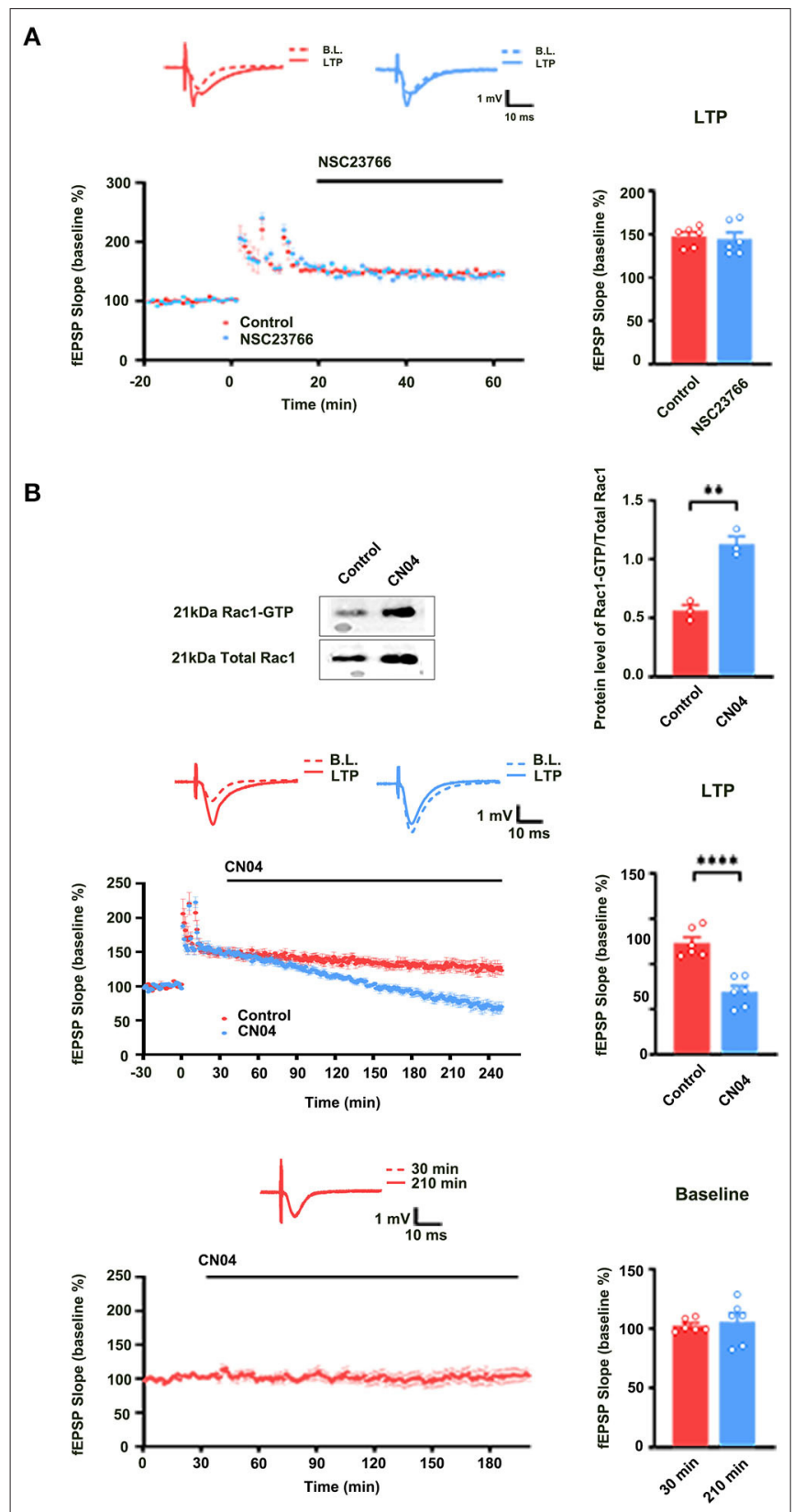

FIGURE 2 | The role of Rac1 in LTP maintenance. (A) Influence of Rac1 antagonist NSC23766 (100 $\mu \mathrm{M})$ on the LTP maintenance (Unpaired $t$-test, $P$ > $0.05, n=6$ in each group). (B) Top: Influence of Rac1 agonist CN04 (424 nM) on Rac1 activation (unpaired $t$-test, ${ }^{* \star} P<0.01, n=3$ in each group). Top right: Average fEPSP slope during the last $15 \mathrm{~min}$ of the LTP recording. Middle: Influence of Rac1 agonist CNO4 (424 nM) on the LTP maintenance (unpaired $t$-test, ${ }^{\star \star \star \star} P<0.0001, n=6$ in each group). Middle right: Average fEPSP slope during the last 15 min of the LTP recording. Bottom: Influence of Rac1 agonist CNO4 (424 nM) on the fEPSP baseline (unpaired $t$-test, $P>0.05, n=$ 6 in each group). All data are shown as mean \pm SEM.

shows the influence of NSC23766 on HFS-induced activation of $\mathrm{PKC} / / \lambda$. We could see that after the application of NSC23766, HFS-induced activation of $\mathrm{PKC} \iota / \lambda$ was inhibited (one-way
ANOVA, $F_{(2,6)}=3.02$, control group, $0.54 \pm 0.043, n=3 ; 30$ min group, $0.69 \pm 0.049, n=3 ; P=0.1233$; Figure $3 B$ ). This result suggests that $\mathrm{Racl}$ activation is an upstream mechanism of the activation of $\mathrm{PKCl} / \lambda$ during the induction phase of LTP.

We further studied how Racl activation results in the activation of $\mathrm{PKC} \iota / \lambda$. We examined the influence of the specific P13K inhibitor LY294002 (Hsueh et al., 2015) on Rac1-induced activation of $\mathrm{PKC} \iota / \lambda$. Firstly, we examined Rac1 agonist CN04induced activation of $\mathrm{PKC} / \lambda$. The rats were divided into five groups:one group was control group where ACSF was applied, and the other four groups were divided into 15-min group, 30-min group, 60-min group, and 90 -min group according to the duration of the application of CN04 (424 nM). Figure 4A shows Rac1 agonist CN04-induced activation of $\mathrm{PKC} / \lambda$ (oneway ANOVA, $F_{(4,15)}=5.899$, control group, $0.72 \pm 0.014, n=4$; 60 -min group, $1.03 \pm 0.073, n=4$, vs. control group; $P=0.0443$; 90 -min group, $1.20 \pm 0.140, n=4$, vs. control group; $P=0.0023$; Figure 4A). This result suggests that CN04 indeed can induce the activation of $\mathrm{PKC} \iota / \lambda$. We then examined the influence of LY294002 on Rac1-induced activation of $\mathrm{PKC} \iota / \lambda$. The rats were divided into three groups:control group where ACSF was applied, CN04 group where CN04 (424 nM) was applied, and CN04 + LY294002 group where CN04 (424 nM) and LY294002 (100 $\mu \mathrm{M})$ were co-applied. Figure 4B shows the influence of LY294002 on Racl-induced activation of $\mathrm{PKC} \iota / \lambda$. We could see that after the application of LY294002, Racl-induced activation of $\mathrm{PKC} \iota / \lambda$ was inhibited (one-way ANOVA, $F_{(2,6)}=16.96$, CN04 group, 1.17 $\pm 0.079, n=3$; LY294002 + CN04 group, $0.77 \pm 0.033, n=$ 3; $P=0.0037$; Figure 4B), whereas LY294002 treatment had no influence on basis of $\mathrm{PKC \iota} / \lambda$ activation (unpaired $t$-test, $t_{(4)}=$ 0.6160 , control group, $0.74 \pm 0.031, n=3$; LY294002 group, 0.71 $\pm 0.028, n=3 ; P=0.5712$; Figure 4C). These results suggest that $\mathrm{Racl}$ activation may result in the activation of $\mathrm{PKC} / / \lambda$ through PI3K pathway in the hippocampus.

\section{Activation of Rac1 During the Maintenance of LTP Results in the Inhibition of PKMS Through LIMK Pathway in the \\ Hippocampus}

Previous study showed that PKM $\zeta$ was a key molecule for the maintenance of LTP (Baltaci et al., 2019). It is unclear whether Racl-induced LTP decay during the maintenance phase is related to the inhibition of the expression of PKM $\zeta$. To address this question, we examined the effect of a specific Rac1 agonist CN04 on increased expression of $\mathrm{PKM} \zeta$ during the maintenance phase of LTP. The rats were divided into three groups:one group was control group where slices were not given HFS, and the other two groups were divided into 60-min group and 120-min group based on the time duration after giving HFS. Figure 5A shows that the expression of $\mathrm{PKM} \zeta$ showed a significant increase at $120 \mathrm{~min}$ after LTP induction (one-way ANOVA, $F_{(2,6)}=14.83$, control group, $0.80 \pm 0.061, n=3 ; 120$ - $m$ in group, $1.52 \pm 0.088, n=3 ; P=$ 0.0031; Figure 5A). Figure 5B shows the influence of CN04 on the increased expression of $\mathrm{PKM} \zeta$ during the maintenance phase of LTP. We could see that after the application of CN04, the expression of PKM $\zeta$ did not change at 60 and $120 \mathrm{~min}$ after LTP 
A
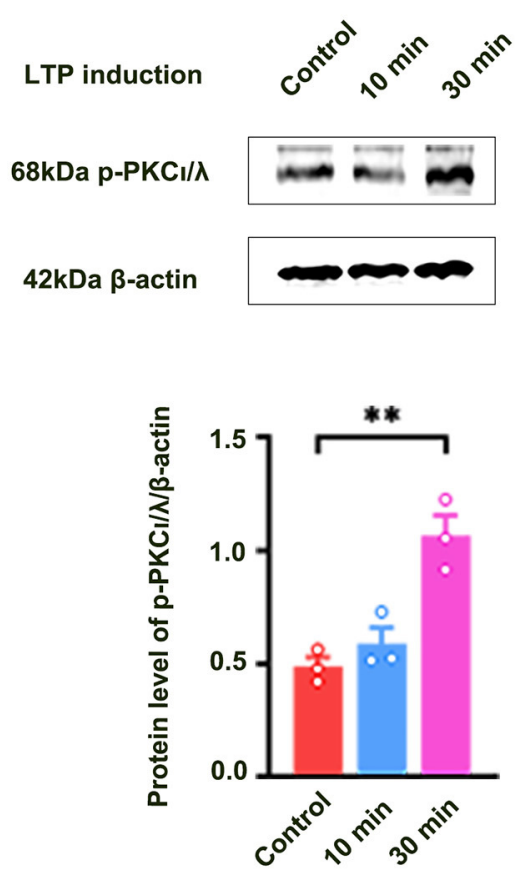

B

NSC23766
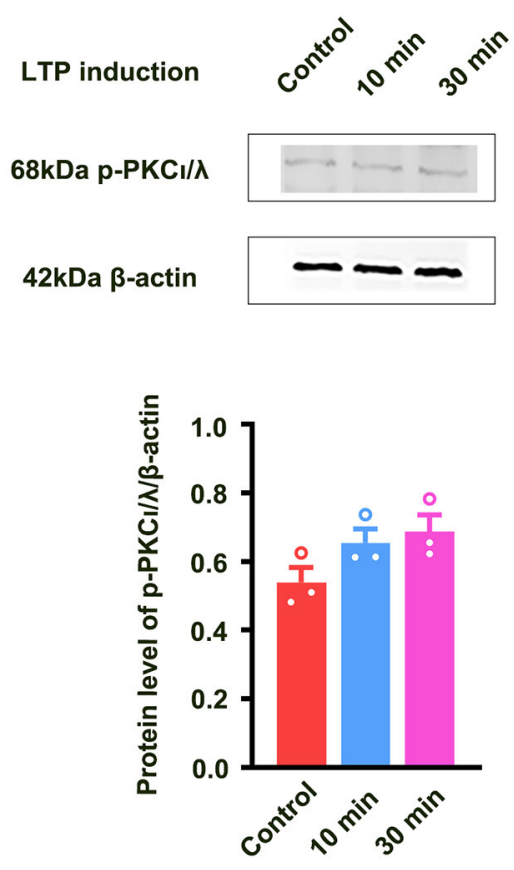

FIGURE 3 | Influence of Rac1 inhibitor NSC23766 on HFS-induced activation of PKCl/ $\lambda$. (A) Influence of three trains of HFS on PKCl/ $\lambda$ activation (one-way ANOVA, ${ }^{\star *} P<0.01, n=3$ in each group). (B) Influence of Rac1 antagonist NSC23766 $(100 \mu \mathrm{M})$ on PKCl/ $\lambda$ activation after giving HFS (one-way ANOVA, $P>0.05, n=3$ in each group). All data are shown as mean \pm SEM.

induction, compared with the control group (one-way ANOVA, $F_{(2,6)}=1.480$, control group, $0.77 \pm 0.045, n=3 ; 120$-min group, $0.96 \pm 0.082, n=3 ; P=0.3002$; Figure 5B). This result suggests that $\mathrm{PKM} \zeta$ is a downstream molecule of Rac1 activation during the maintenance phase of LTP.

We further studied how Rac1 activation results in a decrease in the expression of PKM $\zeta$. We examined the influence of a specific LIMK inhibitor BMS-5 (Lunardi et al., 2018) on Rac1-induced decrease in the expression of $\mathrm{PKM} \zeta$. The rats were divided into three groups:a control group where ACSF was applied, CN04 group where CN04 was applied, and CN04 + BMS-5 group where CN04 and BMS-5 were co-applied. Result showed that after the application of BMS-5, Rac1-induced decrease in the expression of tetanization-induced $\mathrm{PKM} \zeta$ was reversed (one-way ANOVA, $F_{(2,6)}=60.83$, CN04 group, $24.6 \pm 3.5 \%, n=3$; control group, $92.1 \pm 5.8 \%, n=3$, vs. CN04 group $P<0.0001$; BMS$5+\mathrm{CN} 04$ group, $52.8 \pm 3.2 \%, n=3$, vs. CN04 group; $P=0.0067$; Figure 6 bottom right), whereas the BMS- 5 treatment had no influence on the basis of the expression of PKM $\zeta$ (unpaired $t$ test, $t_{(4)}=0.2662$, control group, $0.84 \pm 0.038, n=3$; BMS- 5 group, $0.85 \pm 0.030, n=3 ; P=0.8033$; Figure $6 B$ ). This result suggests that Rac1-induced LTP decay during the maintenance phase is related to the inhibition of the expression of PKM $\zeta$ in the hippocampus.

\section{DISCUSSION}

Previous studies have examined the role of the activation of Rac1 in LTP. Martinez and Tejada-Simon (2011) reported that the induction of LTP in the hippocampus was coupled with the activation of Rac1 in area CA1 and the inhibition of Rac1 suppressed the induction of LTP in a dose-dependent manner (Martinez and Tejada-Simon, 2011). This result is consistent with our current conclusion that Racl participates in the induction of LTP in the hippocampus. This statement was also supported by the findings that upon induction of structural LTP (sLTP) using uncaging of glutamate on single spines, an activation of Racl was observed and the addition of Rac1 inhibitor before sLTP induction effectively inhibited sLTP. However, the statement in this paper that the persistent activation of Racl was required for the maintenance of sLTP still lacked the evidence because the activation of Racl was observed only for $33 \mathrm{~min}$ and not for a longer duration. Moreover, Martinez and Tejada-Simon (2011) reported that the activity of Racl showed a transient increase during the induction of LTP, but returned to the control level during the maintenance phase of LTP (Saneyoshi et al., 2019). This result is consistent with our current statement that the level of Rac1 activity is rather low during the maintenance phase of LTP. This statement is also supported by the result 


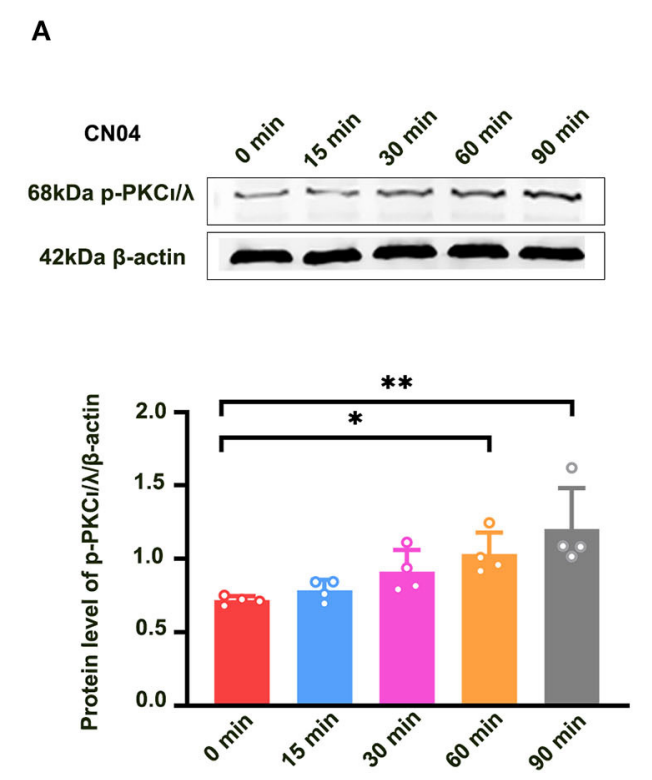

B
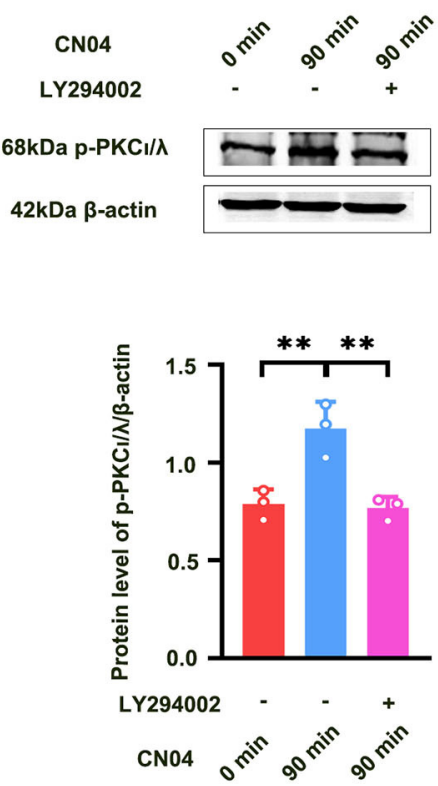

C

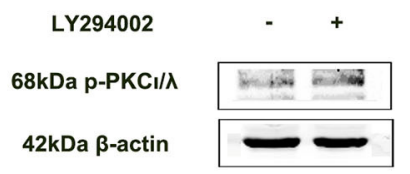

FIGURE 4 | Influence of P13K inhibitor LY294002 on Rac1-induced activation of PKCl/ $\lambda$. (A) Influence of Rac1 agonist CNO4 (424 nM) on PKCl/ $\lambda$ activation (one-way ANOVA, ${ }^{*} P<0.05,{ }^{*} P<0.01, n=4$ in each group). (B) Influence of P13K inhibitor LY294002 (100 $\left.\mu \mathrm{M}\right)$ on the effects of Rac1 agonist CN04 (424 nM) on PKCl/ $\lambda$ activation (one-way ANOVA, ${ }^{* *} P<0.01, n=3$ in each group). (C) Influence of P13K inhibitor LY294002 (100 $\left.\mu \mathrm{M}\right)$ on PKCı/ $\lambda$ activation (unpaired $t$-test, $P>0.05, n=$ 3 in each group). All data are shown as mean \pm SEM.

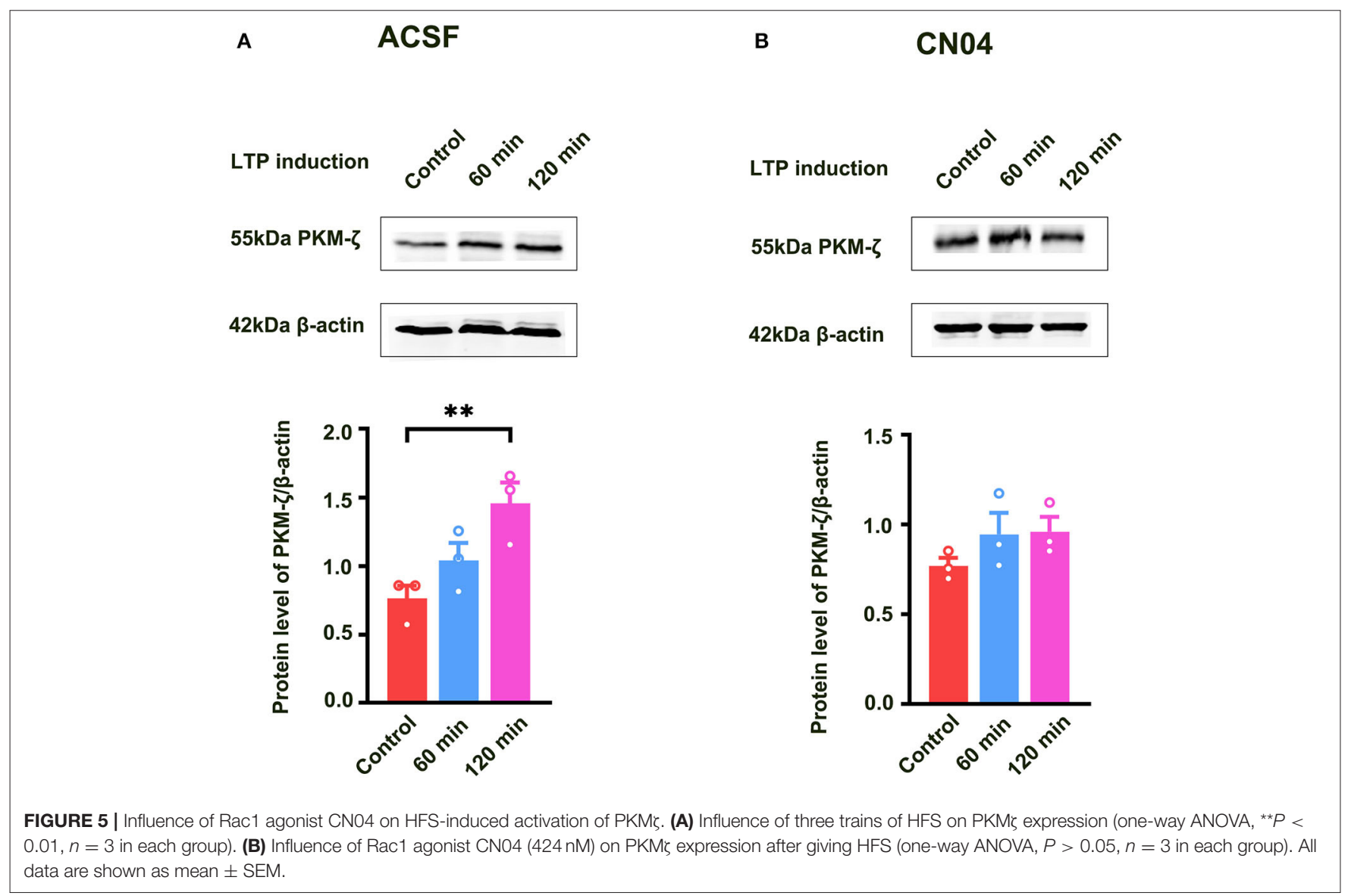


A
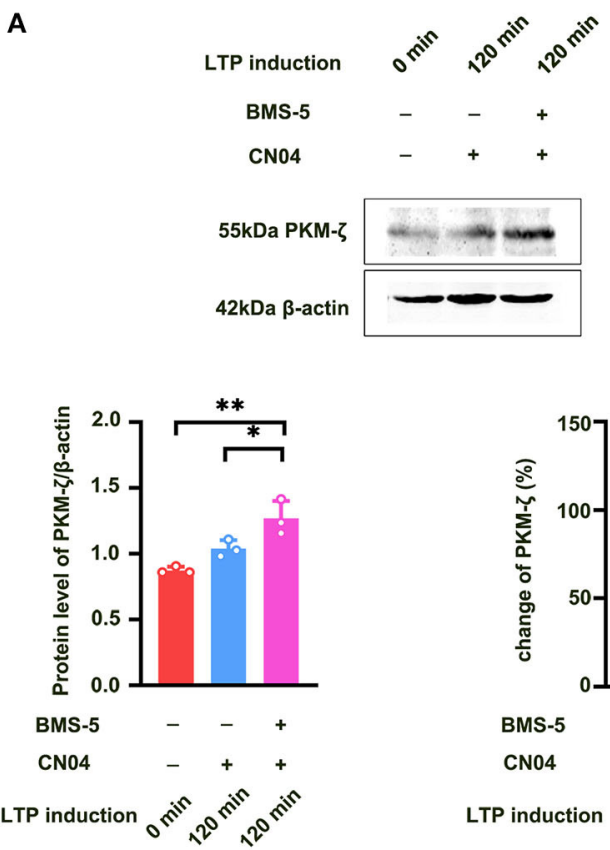

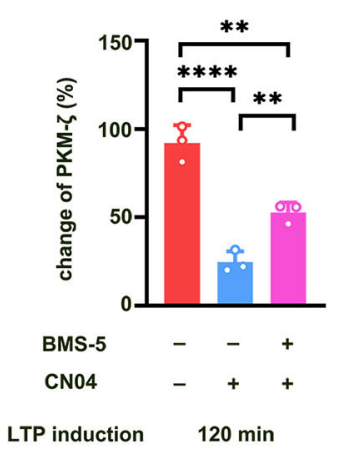

B
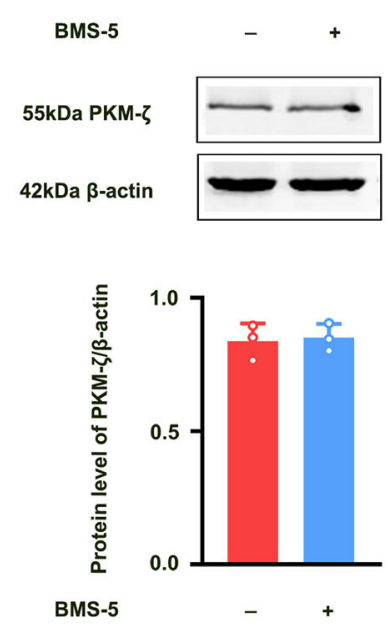

FIGURE 6 | Influence of LIMK inhibitor BMS-5 on Rac1-induced decrease in the expression of PKM . (A) Influence of LIMK inhibitor BMS-5 (100 $\mu$ M) on the effects of Rac1 agonist CNO4 (424 nM) on PKM $\zeta$ expression at 120 min after giving HFS (Bottom left: one-way ANOVA, ${ }^{\star} P<0.05$, ${ }^{\star \star} P<0.01, n=3$ in each group). Bottom right: The bar graph shows the change of PKM $\zeta$ expression from 0 min to 120 min after LTP induction (one-way ANOVA, ${ }^{* \star} P<0.01,{ }^{* \star * *} P<0.0001, n=3$ in each group; The control group data is calculated using the data of Figure 5A). (B) Influence of LIMK inhibitor BMS-5 (100 $\mu M$ ) on PKM $\zeta$ expression (unpaired $t$-test, $P>$ $0.05, n=3$ in each group). All data are shown as mean \pm SEM.

that Rac1 activity is significantly elevated in the hippocampal tissues of adult mice in response to 7 -day social isolation, but decreases to a low level after resocialization (Liu et al., 2018). In contrast, Liu et al. showed that there might be an activation of Racl during the maintenance phase of LTP because after the application of adeno-associated viruses (AAVs) that carried transgene to inhibit endogenous Racl activity, they found that LTP decay during the maintenance phase significantly decreased (Liu et al., 2016). The reason for the difference in Racl activation during the maintenance phase of LTP in different studies remains unknown. However, most of the results from literature (Liu et al., 2016, 2018) and our current study show that the activation of Rac1 during the maintenance phase of LTP in the hippocampus resulted in an accelerated LTP decay. The activator of Racl that we used here was CN04 that could also activate CDC42 and RhoA, and hence the influence of CN04 on LTP maintenance may be due to Rac1, CDC42, or RhoA. In order to determine whether CN04-induced decrease in LTP maintenance was related to Racl activation, we included an experiment on the influence of the inhibition of specific downstream target Pak1 ( $\mathrm{Lv}$ et al., 2013) on CN04-induced decrease in LTP maintenance. The result showed that the Pak1 inhibitor IPA-3 could reverse CN04-induced decrease in LTP maintenance, suggesting that CN04-induced decrease in LTP maintenance was related to Rac1 activation.

It is interesting to study the reason behind why the activation of Racl during the induction and maintenance phases of LTP has an opposing effect in the hippocampus. In a typical downstream signal transduction pathway of the activation of Rac1, Rac1 first activates Pak, which then activates LIMkinase, resulting in the phosphorylation of cofilin, thus inducing an actin polymerization. This pathway partly explains the mechanism underlying the participation of Rac1 in the induction of LTP because Racl-induced actin polymerization results in an enlargement of dendritic spines, which leads to enhanced trapping of AMPA receptors in the postsynaptic membrane and potentiated synaptic transmission (Baltaci et al., 2019). In addition, Tolias et al. (2005) reported that Rac1 could associate with phosphoinositide 3-kinase (PI3K), whereas Ren et al. (2013) reported that LTP induced by PI3K activation could be significantly attenuated by $\mathrm{PKC} \iota / \lambda$ inhibitor $\mathrm{Myr}$ aPKC-PS and the activation of PI3K could activate $\mathrm{PKC} \iota / \lambda$ (Ren et al., 2013). Therefore, it is possible that the activation of Rac1 during the induction phase of LTP leads to an activation of $\mathrm{PKC} \iota / \lambda$ by $\mathrm{PI} 3 \mathrm{~K}$ pathway in the hippocampus. This hypothesis was confirmed by our present result that the inhibition of PI3K could attenuate Rac1-induced activation of $\mathrm{PKC} \iota / \lambda$. Since it has been known that $\mathrm{PKC} \iota / \lambda$ activation is required for both GluA1 phosphorylation and increased surface expression of AMPA receptors during the induction of LTP, it is possible that in addition to Racl-induced Pak-LIMKactin polymerization-enlarged dendritic spines pathway, PI3K$\mathrm{PKC} \iota / \lambda$-GluA1 phosphorylation / increased AMPA receptors may be another downstream signal pathway of the activation of Rac1 to participate in the induction of LTP. In addition, based on the already known upstream signaling pathway of Rac1 
(Saneyoshi et al., 2019), the likely core signaling of early-LTP is: NMDAR to $\mathrm{Ca} 2+$ to CaMKII to $\mathrm{Rac} 1$ to $\mathrm{PI} 3 \mathrm{~K}$ to $\mathrm{PKC} \iota / \lambda$ to AMPA receptors. However, it is hard to explain the opposing effect of the activation of Rac1 on the maintenance of LTP using these two Rac1-induced pathways.

Molecules and signaling pathways mediating the maintenance of LTP have been identified in previous sections (Baltaci et al., 2019). Among them, the continuous enzymatic effect of the constitutively active $\mathrm{PKM} \zeta$ is thought to be the key molecule in the maintenance of LTP (Sacktor, 2011). Wang et al. (2016) reported that after $30 \mathrm{~min}$ of LTP induction, $\mathrm{PKC} / \lambda$ significantly increased but then returned to control level $2 \mathrm{~h}$ after LTP induction, whereas PKM $\zeta$ significantly increased after $2 \mathrm{~h}$ of LTP induction (Wang et al., 2016). In the meantime, LTP inductioninduced increase in the Racl activity also returned to control level during the maintenance phase of LTP (Martinez and TejadaSimon, 2011). Therefore, it appears that Racl does not participate in the maintenance of LTP. However, if exogenous activation of Rac1 during the maintenance phase of LTP, the activated Rac1 could inhibit LTP (Liu et al., 2016, 2018 and present result). Obviously, this inhibition is not due to the re-activation of $\mathrm{PKC} \iota / \lambda$ by $\mathrm{Rac} 1$ because the role of the activation of $\mathrm{PKC} \iota / \lambda$ is to potentiate synaptic transmission. Thus, we proposed that it was possible that the activation of Racl during the maintenance phase of LTP inhibited LTP via the suppression of PKM $\zeta$. This statement was supported by our present result that the activation of Racl during the maintenance phase of LTP could suppress $\mathrm{PKM} \zeta$.

Positive feedback-like mechanism has been proposed to provide PKM $\zeta$ required to maintain LTP (Baltaci et al., 2019). The $\mathrm{PKM} \zeta \mathrm{mRNA}$ is carried to the dendrites following transcription, but its translation is inhibited by a peptidyl-prolyl isomerase PIN1. Following LTP induction, the activity of PIN1 diminishes and its suppressive effect on PKM $\zeta$ translation ceases, and then $\mathrm{PKM} \zeta$ performs synthesis during the maintenance phase of LTP. So there are two possible ways by which the activation of Rac1 inhibits $\mathrm{PKM} \zeta$ : one is the inhibition of $\mathrm{PKM} \zeta$ transcription and the other is the inhibition of PKM $\zeta$ translation. Obviously, the typical downstream signal transduction pathway of Rac1 through LIM-kinase-cofilin-actin cannot explain the inhibitory effect of Racl on PKM $\zeta$ because this pathway is not related to the transcription and translation of proteins. Thus, alternative downstream pathways independent of cofilin-actin of Rac1 should be considered. Yang et al. (2004) reported that LIM-kinase could directly phosphorylate cAMP-responsive element-binding protein (CREB), which led to the stimulation of subsequent gene transcription (Yang et al., 2004) and participated in the maintenance of LTP (Todorovski et al., 2015). Ramos et al. reported that when phospho-CREB was increased in the aged prefrontal cortex, further stimulation of this pathway, even with a very low dose of an activator could exacerbate memory deficits (Ramos et al., 2003). These pieces of evidence suggest that if phospho-CREB already increases, further stimulation of this pathway may accelerate the decay of the maintenance of LTP, which exacerbates memory deficits. This statement was supported by the result that there was an activation of LIM-kinase accompanied by an increase in CREB during the maintenance phase of LTP (Todorovski et al., 2015) and that the further activation of LIM-kinase by Racl during the maintenance phase of LTP accelerated the decay of the maintenance of LTP. However, it is still unclear how over-activated CREB inhibits the transcription or translation of PKM $\zeta$.

In conclusion, the present results showed that the activation of Rac1 during the induction of LTP leads to an activation of $\mathrm{PKC} \iota / \lambda$ by $\mathrm{PI} 3 \mathrm{~K}$, whereas the activation of Racl during the maintenance of LTP leads to the inhibition of PKM $\zeta$ by LIMK in the hippocampus. These results suggest that during different stages of LTP, the activation of Racl can modulate different signaling pathways, which leads to an opposing effect on the induction and maintenance of LTP in the hippocampus.

\section{STAR METHODS \\ Key Resources Table (KRT)}

\begin{tabular}{ll}
\hline REAGENT or & SOURCE \\
RESOURCE & \\
\hline
\end{tabular}

\section{Antibodies}

anti-Rac1

anti- $\beta$-actin

anti-p-PKC $\iota / \lambda$

(Thr555/563)

anti-PKCS (c-20)

Medicine

NSC23766

Rho/Rac/cdc42

Activator I (CNO4)

LY294002

IPA-3

BMS-5

$\begin{array}{ll}\begin{array}{l}\text { Pierce } \\ \text { Santa Cruz } \\ \text { Abcam }\end{array} & \begin{array}{l}\text { Cat\#16118 } \\ \text { Cat\#sc-47778 } \\ \text { Cat\#ab-5813 }\end{array} \\ \text { Santa Cruz } & \text { Cat\#sc-216 } \\ \text { Tocris } & \text { Cat\#2161 } \\ \text { Cytoskeleton } & \text { Cat\#CN04-B } \\ \text { MedchemExpress } & \text { Cat\#9901s } \\ \text { Tocris } & \text { Cat\#3622 } \\ \text { MedchemExpress } & \text { Cat\#HY-18305 }\end{array}$

Experimental Models:

Organisms/Strains
Wildtype Sprague

Dawley rats, male

Software and

Algorithms

Prism 10.7

Adobe Photoshop CS6

Clampfit 10.7

Other

active Rac1 Pull-Down

and Detection Kit

\section{JSJ Biotech N/A}

\section{EXPERIMENTAL MODEL AND SUBJECT DETAILS}

Male adult (6-8 weeks) Sprague-Dawley rats were housed singly in a $12 \mathrm{~h}$ light/dark cycle in a temperature- and humiditycontrolled environment with food and water freely available. All experimental procedures conformed to Fudan University as well as the international guidelines on the ethical use of animals. All 
efforts were made to minimize animal suffering and reduce the number of animals used.

\section{METHOD DETAILS}

\section{Slice Preparation and Electrophysiology}

Hippocampal slices $(400 \mu \mathrm{m})$ were prepared from 8-week-old rats using a vibratome (Leica) (Leutgeb et al., 2003). Slices were incubated in $32^{\circ} \mathrm{C}$ oxygenated artificial cerebrospinal fluid (ACSF) containing $124 \mathrm{mM} \mathrm{NaCl}, 3 \mathrm{mM} \mathrm{KCl}, 1.25 \mathrm{mM} \mathrm{KH}_{2} \mathrm{PO}_{4}$, $1 \mathrm{mM} \mathrm{MgSO} 4,2 \mathrm{mM} \mathrm{CaCl}, 26 \mathrm{mM} \mathrm{NaHCO} 3$, and $10 \mathrm{mM}$ glucose $(\mathrm{pH}$ 7.2-7.4) for at least $2 \mathrm{~h}$ before recording. Slices were placed in a recording chamber and perfused by oxygensaturated ACSF with a flow rate of $4-5 \mathrm{ml} / \mathrm{min}$. Extracellular field excitatory post-synaptic potentials (fEPSPs) in the Schaffer Collateral pathway were synaptically evoked at $0.017 \mathrm{~Hz}$ and recorded in the CA1 region. The fEPSPs were evoked using a stimulation intensity that elicited a $40 \%$ maximal response. LTP was induced by three trains of high-frequency stimulation (HFS, $1 \mathrm{~s}$ at $100 \mathrm{~Hz}$ spaced $5 \mathrm{~min}$ apart). The stimulation during the HFS was the same strength as test stimulation. The fEPSPs were recorded with an Axopatch700B amplifier (Axon) connected to a Digidata1440 interface (Axon). Data acquisition and analysis were performed using the Axon software packages Clampfit.

\section{Western Blot Analysis}

Four brain slices containing the hippocampal CA1 region were homogenized in a buffer containing $100 \mathrm{mM}$ Tris- $\mathrm{HCl}(\mathrm{pH}=6.7)$, $1 \%$ SDS, $143 \mathrm{mM} 2$-mercaptoethanol, and 1\% protease inhibitor. The lysate was centrifuged at $12,000 \mathrm{rpm}$ for $10 \mathrm{~min}$ at $4^{\circ} \mathrm{C}$. The samples were treated with the SDS sample buffer at $100^{\circ} \mathrm{C}$ for $10 \mathrm{~min}$, loaded on a $10 \%$ SDS polyacrylamide gel, and blotted to a nitrocellulose $(\mathrm{NC})$ membrane. The membranes were blocked for $1 \mathrm{~h}$ at room temperature in a blocking solution (Beyotime, China), followed by incubation overnight at $4^{\circ} \mathrm{C}$ with various primary antibodies that included anti-Racl at a dilution of 1:500; anti-pPKC $\iota / \lambda$, anti-PKM $\zeta$, and anti- $\beta$-actin at a dilution of 1:1,000. Afterward, the membranes were rinsed with $1 \times$ TBST (Sangon, China) for three times ( $5 \mathrm{~min}$ for each wash), followed by incubation respectively with IRDye 680 LT goat anti-rabbit secondary antibody $(1: 10,000)$ and IRDye $800 \mathrm{CW}$ goat anti-mouse secondary antibody $(1: 10,000)$ for $1 \mathrm{~h}$ at room temperature. Finally, after rinsing the membranes for three times ( 5 min for each wash) with $1 \times$ TBST, we acquired the images with LI-COR Odyssey system.

\section{Assay for GTPase Activity}

Active Rac1 pull-down was performed as described by the commercial active Rac1 Pull-Down and the Detection Kit protocol (Pierce, catalog \#16118). Briefly, lysates of the rat hippocampal CA1 tissue were centrifuged at $16,000 \mathrm{~g}$ at $4^{\circ} \mathrm{C}$ for $15 \mathrm{~min}$, and then the supernatants were transferred to a new tube, and GTPYS or GDP was added and incubated at $30^{\circ} \mathrm{C}$ for 15 min under the condition of constant agitation. The mixtures were then incubated with glutathione resin beads and glutathione S-transferase-fused Rac-binding domain of p21-activated kinase (Pak) at $4^{\circ} \mathrm{C}$ for $1 \mathrm{~h}$; the beads had been washed several times previously to remove nonspecific binding. The beads and proteins bound to the fusion protein were washed three times with wash buffer at $4^{\circ} \mathrm{C}$, eluted in SDS sample buffer, and analyzed for bound Rac1 by Western blotting using anti-Rac1.

\section{QUANTIFICATION AND STATISTICAL ANALYSIS}

Statistical significance was determined using unpaired $t$-test for comparisons between two groups or ANOVAs for comparisons among three or more groups. All of the statistical details of the experiments can be found in the results. In all cases, $n$ refers to the number of animals. Graphpad Prism 8.4 was used to process and analyze data and make statistical graphs. Data are presented as mean \pm SEM.

\section{DATA AVAILABILITY STATEMENT}

The original contributions presented in the study are included in the article/Supplementary Material, further inquiries can be directed to the corresponding authors.

\section{ETHICS STATEMENT}

The animal study was reviewed and approved by The Animal Care and Use Committee of Shanghai Medical College of Fudan University.

\section{AUTHOR CONTRIBUTIONS}

DC was responsible for conception and design, acquisition of data, analysis, interpretation of data, and drafting or revising the article. XJ, MC, HS, DS, LY, XG, and YW were responsible for the acquisition of data and analysis and interpretation of data. BL was responsible for conception and design and analysis and interpretation of data. PZ was responsible for conception and design, analysis and interpretation of data, and drafting or revising the article. All authors contributed to the article and approved the submitted version.

\section{FUNDING}

This study was supported by a Project of the Foundation of National Natural Science of China (32030051, 31970956).

\section{SUPPLEMENTARY MATERIAL}

The Supplementary Material for this article can be found online at: https://www.frontiersin.org/articles/10.3389/fnmol. 2021.720371/full\#supplementary-material

Supplementary Figure 1 | Influence of Pak1 inhibitor IPA-3 on CNO4-induced decrease of LTP maintenance.Influence of adding Pak1 inhibitor IPA-3 (100 $\mu \mathrm{M})$ before the application of CNO4 (424 nM) on the CNO4-induced decrease of LTP maintenance (unpaired $t$-test, ${ }^{* *} P<0.01, n=6$ in each group). All data are shown as mean \pm SEM.

Supplementary Figure 2 | The full-length immunoblot. 


\section{REFERENCES}

Baltaci, S. B., Mogulkoc, R., and Baltaci, A. K. (2019). Molecular mechanisms of early and late LTP. Neurochem. Res. 44, 281-296. doi: 10.1007/s11064-018-2695-4

Hall, A. (2005). Rho GTPases and the control of cell behaviour. Biochem. Soc. Trans. 33, 891-895. doi: 10.1042/BST0330891

Hsueh, Y. J., Chen, H. C., Wu, S. E., Wang, T. K., Chen, J. K., and Ma, D. H. (2015). Lysophosphatidic acid induces YAP-promoted proliferation of human corneal endothelial cells via PI3K and ROCK pathways. Mol. Ther. Methods Clin Dev 2:15014. doi: $10.1038 / \mathrm{mtm} .2015 .14$

Jiang, L., Mao, R., Tong, J., Li, J., Chai, A., Zhou, Q., et al. (2016). Inhibition of Racl activity in the hippocampus impaired extinction of contextual fear. Neuropharmacology 109, 216-222. doi: 10.1016/j.neuropharm.2016.06.017

Leutgeb, J. K., Frey, J. U., and Behnisch, T. (2003). LTP in cultured hippocampalentorhinal cortex slices from young adult (P25-30) rats. J. Neurosci. Methods 130, 19-32. doi: 10.1016/S0165-0270(03)00228-0

Liu, Y., Du, S., Lv, L., Lei, B., Shi, W., Tang, Y., et al. (2016). Hippocampal Activation of Racl Regulates the Forgetting of Object Recognition Memory. Curr. Biol. 26, 2351-2357. doi: 10.1016/j.cub.2016.06.056

Liu, Y., Lv, L., Wang, L., and Zhong, Y. (2018). Social isolation induces racl-dependent forgetting of social memory. Cell Rep. 25, 288-295. doi: 10.1016/j.celrep.2018.09.033

Lunardi, P., Sachser, R. M., Sierra, R. O., Pedraza, L. K., Medina, C., de la Fuente, V., et al. (2018). Effects of hippocampal LIMK inhibition on memory acquisition, consolidation, retrieval, reconsolidation, and extinction. Mol. Neurobiol. 55, 958-967. doi: 10.1007/s12035-016-0361-x

Luo, L. (2000). Rho GTPases in neuronal morphogenesis. Nat. Rev. Neurosci. 1, 173-180. doi: 10.1038/35044547

Lv, Z., Hu, M., Zhen, J., Lin, J., Wang, Q., and Wang, R. (2013). Rac1/PAK1 signaling promotes epithelial-mesenchymal transition of podocytes in vitro via triggering beta-catenin transcriptional activity under high glucose conditions. Int. J. Biochem. Cell Biol. 45, 255-264. doi: 10.1016/j.biocel.2012.11.003

Martinez, L. A., and Tejada-Simon, M. V. (2011). Pharmacological inactivation of the small GTPase Rac1 impairs long-term plasticity in the mouse hippocampus. Neuropharmacology 61, 305-312. doi: 10.1016/j.neuropharm.2011.04.017

Ramos, B. P., Birnbaum, S. G., Lindenmayer, I., Newton, S. S., Duman, R. S., and Arnsten, A. F. (2003). Dysregulation of protein kinase a signaling in the aged prefrontal cortex: new strategy for treating agerelated cognitive decline. Neuron 40, 835-845. doi: 10.1016/S0896-6273(03)0 0694-9

Ren, S. Q., Yan, J. Z., Zhang, X. Y., Bu, Y. F., Pan, W. W., Yao, W., et al. (2013). PKC lambda is critical in AMPA receptor phosphorylation and synaptic incorporation during LTP. EMBO J. 32, 1365-1380. doi: 10.1038/emboj. 2013.60

Sacktor, T. C. (2011). How does PKM zeta maintain long-term memory? Nat. Rev. Neurosci. 12, 9-15. doi: 10.1038/nrn2949

Saneyoshi, T., Matsuno, H., Suzuki, A., Murakoshi, H., Hedrick, N. G. Agnello, E., et al. (2019). Reciprocal activation within a kinase-effector complex underlying persistence of structural LTP. Neuron 102, 1199-1210. doi: 10.1016/j.neuron.2019.04.012

Todorovski, Z., Asrar, S., Liu, J., Saw, N. M., Joshi, K., Cortez, M. A., et al. (2015). LIMK1 regulates long-term memory and synaptic plasticity via the transcriptional factor CREB. Mol. Cell. Biol. 35, 1316-1328. doi: 10.1128/MCB.01263-14

Tolias, K. F., Bikoff, J. B., Burette, A., Paradis, S., Harrar, D., Tavazoie, S., et al. (2005). The Rac1-GEF Tiam1 couples the NMDA receptor to the activitydependent development of dendritic arbors and spines. Neuron 45, 525-538. doi: 10.1016/j.neuron.2005.01.024

Wang, S., Sheng, T., Ren, S., Tian, T., and Lu, W. (2016). Distinct Roles of PKCiota/lambda and PKMzeta in the Initiation and Maintenance of Hippocampal Long-Term Potentiation and Memory. Cell Rep. 16, 1954-1961. doi: 10.1016/j.celrep.2016.07.030

Yang, E. J., Yoon, J. H., Min, D. S., and Chung, K. C. (2004). LIM kinase 1 activates cAMP-responsive element-binding protein during the neuronal differentiation of immortalized hippocampal progenitor cells. J. Biol. Chem. 279, 8903-8910. doi: 10.1074/jbc.M311913200

Conflict of Interest: The authors declare that the research was conducted in the absence of any commercial or financial relationships that could be construed as a potential conflict of interest.

Publisher's Note: All claims expressed in this article are solely those of the authors and do not necessarily represent those of their affiliated organizations, or those of the publisher, the editors and the reviewers. Any product that may be evaluated in this article, or claim that may be made by its manufacturer, is not guaranteed or endorsed by the publisher.

Copyright (c) 2021 Cui, Jiang, Chen, Sheng, Shao, Yang, Guo, Wang, Lai and Zheng. This is an open-access article distributed under the terms of the Creative Commons Attribution License (CC BY). The use, distribution or reproduction in other forums is permitted, provided the original author(s) and the copyright owner(s) are credited and that the original publication in this journal is cited, in accordance with accepted academic practice. No use, distribution or reproduction is permitted which does not comply with these terms. 